\title{
Titrations of Bases with Diphenyl Phosphate in Some Aqueous and Nonaqueous Solvents
}

\author{
Marion Maclean Davis and Hannah B. Hetzer
}

\begin{abstract}
Successful titrations of various bases with diphenyl phosphate in benzene, methanol, water, and mixtures of these solvents have been performed, in some cases potentiometrically (using the glass, saturated calomel electrode-system) and in other cases using indicator dyes. Several of the dyes (bromophthalein magenta E, 5-phenyl-9-diethyl Nile blue, and the anhydro-base of tamarack green and Victoria blue B) have not been used elsewhere as acidbase indicators. Diphenyl phosphate is shown to be a strong acid which is easily obtained and maintained as anhydrous crystals. It is not strong enough, however, to serve as a replacement for perchloric acid in titrations in acetic acid. Attention is called to the differing behavior of benzoic acid and diphenyl phosphate when the solvent is changed from water to methanol. Using a "nonleveling" solvent in place of water makes it possible to obtain separate, sharp end-points in titrating a mixture of two such acids. Other pertinent information about diphenyl phosphate is summarized.
\end{abstract}

\section{Introduction}

As previously explained $[1]^{2}$, we are engaged in developing methods for measuring acidity and basicity in organic solvents, with special emphasis on so-called "inert" solvents. Two essential parts of this research are (1) locating or preparing new chemical tools - indicator dyes and materials suitable for use as standard acids or bases in such solvents; and (2) finding out, by systematic studies, the behavior of different types of acids and bases in various nonaqueous solvents. This paper deals with a study of some of the properties of diphenyl phosphate, $\left(\mathrm{C}_{6} \mathrm{H}_{5} \mathrm{O}\right)_{2} \mathrm{P}(\mathrm{O}) \mathrm{OH}$, with particular attention to its use in titrations with bases in several aqueous and nonaqueous solvents.

Interest in diphenyl phosphate first arose upon noticing in a catalog of chemicals available for experimental purposes [2] that, besides giving a strongly acid solution in water, it is easily soluble in a variety of organic solvents, including benzene and carbon tetrachloride. The interest increased after finding that the acid can be obtained and maintained as pure, anhydrous crystals without difficulty. Very few studies have been made of the comparative effects of aqueous and nonaqueous solvents on the behavior of strong acids, and diphenyl phosphate appeared suitable for such studies, as well as a potentially useful standard acid for nonaqueous titrations. Hitherto, picric acid and a few arylsulfonic acids have been the only commonly available strong, crystalline acids soluble in both water and a variety of organic solvents, and these acids possess certain shortcomings. For instance, picric acid is somewhat hazardous to handle unless 10 percent or so of water has been added, and the yellow color of its salts is sometimes objectionable. A further incentive to studying diphenyl phosphate is the meagerness of the existing knowledge of the

1 This research was supported in part by the United States"Air Force, through the Office of Scientific Research of the Air Research and Development Command.

${ }_{2}^{2}$ Figures in brackets indicate the literature references at the end of this paper acidic behavior of this compound and of various related diaryl phosphates, even though they were first synthesized about 100 years ago and scattered articles have appeared dealing with such topics as their preparation, physical properties, and possible uses.

\section{Apparatus and Miscellaneous Materials}

\subsection{Apparatus}

A titration apparatus similar to the one described in [3], employing a glass and a sleeve-type saturated calomel electrode, was used in the potentiometric titrations. During titrations the temperature was usually within the range $25^{\circ} \pm 2^{\circ} \mathrm{C}$. In most cases $10-\mathrm{ml}$ burets having $0.05-\mathrm{ml}$ graduations were used.

Melting points were determined by the capillarytube method, using an automatically-stirred, electrically-heated, high-boiling silicone oil and an ASTM thermometer maintained at 3-inch immersion.

\subsection{Solvents}

The organic solvents were all of a grade that was claimed to meet ACS specifications. Acetic acid and methanol were said to contain approximately 0.5 percent of water. Distilled water was boiled shortly before use, to remove carbon dioxide.

\subsection{Bases}

1,3-Diphenylguanidine was recrystallized twice from toluene and once from benzene-cyclohexane; the melting point was $149.0^{\circ}$ to $149.8^{\circ} \mathrm{C}$. Titration with perchloric acid in glacial acetic acid indicated a purity of at least 99.5 percent. Di-o-tolylguanidine was recrystallized twice from 95-percent ethanol; melting point, $175.8^{\circ}$ to $176.3^{\circ} \mathrm{C}$. Titration in aqueous alcohol with $0.1 \mathrm{~N}$ hydrochloric acid indicated its purity to be 99.9 percent; titration in glacial acetic acid gave the value 99.6 percent. A commercial high grade of guanidine acetate was used without further treatment. Commercial high grad 
tribenzylamine was recrystallized from ethanol; melting point, $92.8^{\circ}$ to $93.2^{\circ} \mathrm{C}$. A good grade of triethylamine was distilled from sodium hydroxide immediately before use; the middle fraction was used. Tris (hydroxymethyl)aminomethane was part of a purified sample that had been used in the determination of the aqueous dissociation constant [4].

Potassium acid phthalate was NBS Standard Sample for acidimetry. Sodium acetate in glacial acetic acid was prepared by dissolving anhydrous sodium carbonate of analytical grade. The solutions containing sodium methylate were prepared from the solid compound.

\subsection{Indicator Dyes}

Both acidic and basic dyes were used in the titrations. Bromophthalein magenta E (fig. 1, formula I, $\mathrm{X}=\mathrm{COOC}_{2} \mathrm{H}_{5}$ ) was prepared at the Bureau; its application in some nonaqueous acid-base titrations was described previously [1]. ${ }^{3}$ The other acidic indicator dyes-phenolphthalein, p-naphtholbenzein $(\alpha$-naphtholbenzein), and the various sulfonephthaleins were all commercial materials; the sulfonephthaleins were used as their sodium salts.

Most basic indicator dyes are supplied commercially as a salt (generally, the chloride). Azo dyes, however, are often available only as the free base, though sometimes also available as a salt. For reasons of solubility, the salt is more convenient for aqueous titrations, and the free base is necessary for titrations in benzene and similar solvents. Either form of the dye dissolves readily in alcohols. In the work described here all basic dyes were used as the free bases with the exception of $\alpha$-Naphthyl red (4-phenylazo-1-naphthylamine hydrochloride) and the three Nile blues.

p-Ethoxychrysoidin, 4-(p-ethoxyphenylazo)-mphenylenediamine, was isolated by adding an excess of 10-percent sodium hydroxide to a hot solution of

3 This indicator is now obtainable from Distillation Products Industries, listed as Eastman Organic Chemical No. 6810 (tetrabromophenolphthalein ethyl ester).
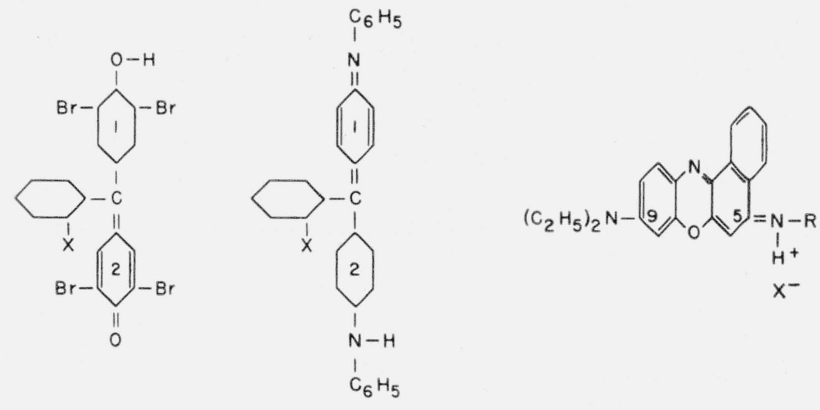

I

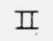

III

Figure 1. Formulas of indicators.

I. Bromophthalein magenta E (tetrabromophenolphthalein ethyl ester); $\mathrm{X}=\mathrm{COOC}_{2} \mathrm{H}_{5}$.

Treen anhydro-base (anhydro-N, $\mathrm{N}^{\prime}$-diphenyl- $p, p^{\prime}$-diamino- $o^{\prime \prime}$ chlorotriphenylcarbinol); $\mathrm{X}=C \mathrm{C}$.

III. 5-Phenyl-9-diethyi Nile blue (5-phenylimino-9-diethylaminobenzo[a]phenoxazonium chloride); $\mathrm{X}=C 1$. the commercial hydrochloride in 95-percent ethanol, then pouring the ethanolic solution into water; the base, consisting of fluffy yellow needles, was washed with water and dried at room temperature, first in the air and then in a vacuum desiccator containing anhydrous alumina.

For part of the work the indicator dyes commercially available were not suitable, and three new basic indicators were used. These call for some additional comment.

\section{a. Tamarack Green Anhydro-Base}

Tamarack green anhydro-base (fig. 1, formula II, $\mathrm{X}=\mathrm{Cl}$ ) has not been described previously, although the parent compound $(\mathrm{X}=\mathrm{H})$ has been known for many years. Both compounds belong to a class of derivatives of triphenylmethane, known as "Homolka bases" or "anhydro-bases", the first one of which was prepared by Homolka; later this class of compounds was studied by other workers, notably by Baeyer and Villiger $[5,6] .{ }^{4}$ The parent compound was first prepared by Meldola, who prepared its hydrochloride by the reaction of diphenylamine with benzotrichloride in the presence of anhydrous, powdered zinc chloride and named it "diphenylamine green" [7]. An impure specimen of the hydrochloride which he had prepared earlier by the oxidizing action of air on a mixture of diphenylamine and benzyl chloride had been introduced into commerce under the name "viridine." Meldola did not isolate the pure anhydro-base, although he observed the red-brown color, due to its presence, which resulted when a solution of the salt was shaken with benzene and sodium hydroxide solution. In later, more complete studies, Baeyer and Villiger obtained the anbydrobase in an analytically pure condition; they applied the name "viridine" to the pure dye [5].

Tamarack green hydrochloride was prepared with the substitution of 0 -chlorobenzotrichloride for benzotrichloride, but otherwise essentially as described by Meldola [7]. ${ }^{6}$ The hydrochloride was converted to the picrate which, in turn, was converted to the anhydro-base, as outlined in [5], except for the use of benzene in place of ether. The properties of tamarack green (both hydrochloride and free base) resemble closely those described for the parent compounds $[5,7,8]$.

4 These seldom-mentioned compounds give yellow to red solutions in solvents like benzene. The nitrogen atom attached to ring 1 (see formula II) is rather strongly basic, and on the addition of a proton forms a resonant cation that is deeply colored (red to green, depending on the substituents on the nitrogens and in the rings). (This is a simplified discussion; it is probably not correct to assume that in solvents like benzene the proton is completely detached from the anion of the acid molecule of which it was originally a part.) Anhydro-bases tend to add water to form the well-known colorless compounds called "carbinol bases" or "color bases." Alcohols, ammonia, and primary or secondary amines give similar colorless compounds, $\mathrm{H}$ adding to the nitrogen of ring 1 , and the rest of the molecule $\left(-\mathrm{OH},-\mathrm{OR}\right.$, or $\left.-\mathrm{NR}_{2}\right)$ becoming linked to the methane carbon atom. A similar tendency is responsible for the fading by alkali of fabrics dyed with triphenylmethane dyes, but it has been reduced by introducing a bulky substituent (for instance, $\mathrm{Cl}, \mathrm{NO}_{2}$, or $\mathrm{SO}_{3} \mathrm{H}$ ) in an $o^{\prime \prime}$-position [6]. '

$0^{\prime \prime}-\mathrm{Cl}$ in tamarack green was introduced with such an objective in mind.

5 Hill, Branch, and Patapoff have also prepared and studied the anhydro-base and called it "viridine" [8].

6 The dye was prepared almost 10 years ago by M. M. Davis and P. J. Schuhmann, for exploratory studies. The base was not analyzed and may have contained a little carbinol. Since preparation, its behavior as an indicator has not visibly changed. 


\section{b. Victoria Blue Anhydro-Base}

Comparison of the formula of Victoria blue $B$ anhydro-base, $\left.\mathrm{C}_{6} \mathrm{H}_{5} \mathrm{~N}=\mathrm{C}_{10} \mathrm{H}_{6}=\mathrm{C}_{[} \mathrm{C}_{5} \mathrm{H}_{4} \mathrm{~N}\left(\mathrm{CH}_{3}\right)_{2}\right]_{2}$, with that of tamarack green anhydro-base reveals several differences in their structures, but it can be seen that they should function similarly when used as acid-base indicators. Victoria blue B anhydrobase was first prepared by Nathansohn and Müller [9], who erroneously believed it to be the carbinol. Noelting and Saas, after later studies [10], reported that the anhydro-base cannot be converted into the carbinol or to a carbinol ether or corresponding amino derivative. ${ }^{7}$

To prepare the anhydro-base, $4 \mathrm{~g}$ of the commercial salt (Color Index No. 729) was dissolved in about $800 \mathrm{ml}$ of hot water, the solution was filtered to remove insoluble material, and the filtrate was stirred mechanically while 2 to $3 \mathrm{ml}$ of $6 N$ sodium hydroxide was added. The dark brown precipitate was filtered and washed with the aid of suction, air-dried, and then recrystallized from benzene

\section{c. 5-Phenyl-9-diethyl Nile Blue (5-Phenylimino-9-diethylamino- benzo[a]phenoxazonium Chloride, $\mathrm{C}_{26} \mathrm{H}_{24} \mathrm{ClN}_{3} \mathrm{O} \cdot \mathrm{H}_{2} \mathrm{O}$ )}

The general structure of the three Nile blues used in the work is shown as formula III in figure 1 . The only important difference among them is in the group R, which is hydrogen in Nile blue A (CI No. 913), benzyl in Nile blue 2B (CI No. 914), and phenyl in 5-phenyl-9-diethyl Nile blue. All of the salts give blue solutions which turn red (pink, if dilute) when the free base is liberated. As would be expected, the free base is strongest when $\mathrm{R}$ equals $\mathrm{H}$ and weakest when $\mathrm{R}$ equals phenyl. ${ }^{8}$

Phenyl diethyl Nile blue was a very pure material prepared in the research laboratories of American Cyanamid Company [12]. ${ }^{9}$

\section{Diphenyl Phosphate}

\subsection{General Discussion}

Diphenyl phosphate has been studied intermittently since 1866 . It was first detected by Hlasiwetz and Grabowski in a mixture formed by the reaction of phenol with phosphorus pentoxide [13], and was first isolated, as a brown oil, by Glutz, after treatment of phenol with phosphorus pentachloride [14]. Next, Jacobsen studied the stepwise reaction of phenol with phosphorus oxychloride, followed by hydrolysis of the intermediate products, and thus obtained crystalline monophenyl and tri-

Evidently a naphthyl group, like an $o^{\prime \prime}$-substituted phenyl group, impedes the addition of a fourth group to the methane carbon atom of triphenylmethane dyes.

8 See figure 5 and related discussion. The $p H$ range of the commercial Nile blue A used is given as about 10.2 (blue) to 13.1 (purple), and that of the commercial Nile blue $2 \mathrm{~B}$, as about 7.2 to 8.6 . (However, Kolthoff reported the $p \vec{H}$ range of Nile blue A to be 9.0 (blue) to 10.4 (red) [11].)

9 Phenyl diethyl Nile blue is one of a series of benzophenoxazine dyes prepared for possible chemotherapeutic applications and was made available, through the courtesy of J. J. Denton, for investigations of its utility as an acid-base indicator in nonaqueous media. As Dr. Denton has pointed out in correspondence, the compounds known commercially as Nile blue dyes are dye mixtures, whereas the specimen of 5-phenyl-9-diethyl Nile blue supplied was analytically pure, and is therefore properly referred to by its strict chemical name. However, a shorter name is needed for common usage, and the name used in this paper was agreed on. For ordinary titrations a less pure material should, of course, be satisfactory. phenyl phosphates; diphenyl phosphate was obtained only as a colorless, viscous oil that would not solidify in a freezing mixture [15].

Rapp, repeating Jacobsen's procedure, succeeded in obtaining crystalline diphenyl phosphate by seeding an aqueous solution with a little sodium diphenyl phosphate, and reported the melting point of the acid as $56^{\circ} \mathrm{C}$ [16]. Autenrieth used a slight modification of the Jacobsen procedure and reported that diphenyl phosphate is easily obtained in the crystalline state if not contaminated with phenol; his final product, obtained from an aqueous solution as shimmering leaflets, had the melting point $61^{\circ}$ to $63^{\circ}$ - a value several degrees higher than that of Rapp [17]. The mystery of the divergent melting points was cleared up by Hoeflake's observation that diphenyl phosphate has two crystalline forms, one being anhydrous and the other, a dihydrate [18]. The products of Rapp and Autenrieth were presumably different mixtures of the two forms. Hoeflake's anhydrous diphenyl phosphate was obtained by heating the crude acid (prepared by Jacobsen's method) in vacuo to $60^{\circ}$ to $80^{\circ}$ to remove water of hydration, followed by solution of the acid in cold chloroform, then reprecipitation with ligroin. She described the anhydrous acid as fine needles melting at $70^{\circ}$. The dihydrate was obtained as small leaflets, melting at $51^{\circ}$, by recrystallizing the anhydrous acid from water. Hoeflake furthermore stated that anhydrous diphenyl phosphate takes up two molecules of water in the air, but loses this water again at $30^{\circ}$, also over sulfuric acid. Bernton [19] concurred in Hoeflake's description of the two crystalline modifications of diphenyl phosphate, but reported that the hydrate is very stable in the air.

Various practical applications have been suggested for diphenyl phosphate. Bernton found that it combines in 1:1 ratio with different types of bases (ammonia, methylamine, diethylamine, aniline, amino acids), forming well-crystallized salts [19]. Diphenyl phosphate, together with other diaryland monoaryl phosphates, has been tested as a possible reagent for the determination of iron; aryl phosphates of various metals were prepared [20]. Potassium diphenyl phosphate, like the potassium salts of some related acids, increases the solubilities of various organic compounds in water [21]. Diphenyl phosphate has been used as a substrate in studies of enzvme action, and potassium salts of closely related acids have been reported to destroy certain kinds of bacteria. In industrial applications, diphenyl phosphate has been reported to retard the corrosion of steel sheets, improve lubricating oil for steel-light metal bearings, and excel dibutyl phthalate in reducing the permeability of ethylcellulose to water.

There is no evidence in the literature, or from our experience in using diphenyl phosphate, that it is toxic. However, considering that certain commercial esters of phosphoric acid and pyrophosphoric acid, as well as some related compounds, are known to interfere with normal physiological reactions, it seems prudent to avoid contact of diphenyl phosphate with the skin and inhalation of fine particles. 


\subsection{Purification}

\section{a. Anhydrous Diphenyl Phosphate}

"Anhydrous" diphenyl phosphate was supplied by the Dow Chemical Company, through the kindness of C. W. Robertson. The material as received contained a little water of hydration, was slightly tinged with pink, and had a faint odor of phenol. Diphenyl phosphate is fairly soluble in hot cyclohexane; the amount of cyclohexane needed can be reduced a good deal by adding 10 percent or so of benzene. Any water present in the sample is removed if the cyclohexane or cvclohexane-benzene solution is boiled for a while. The pink material goes into solution and tends to remain with the diphenyl phosphate, but it can be removed by repeated digestions with decolorizing carbon. A good way to remove most of the pink contaminant is to add less than the amount of hot solvent needed for complete solution of the acid; the bulk of the solution is then nearly colorless, the undissolved acid forming a pink oil which adheres to the bottom of the vessel when the colorless portion is decanted off. When the cooled solution is seeded, the acid quickly crystallizes in colorless needles; without seeding, it usually separates first as an oil. The purified acid has no odor. The purest product obtained in our work melted to a colorless liquid at $68.5^{\circ}$ to $69.0^{\circ} \mathrm{C}$, with slight softening from about $68.0^{\circ}$; the untreated acid melted about a degree lower, yielding a pale pink liquid. Titrations in water (see section 4.1) indicated that the best anhydrous acid was about 99.9 percent pure (see also section 4.5).

The crystals of anhydrous diphenyl phosphate showed no tendency to pick up water at room temperature (which was usually in the range $20^{\circ}$ to $\left.25^{\circ} \mathrm{C}\right)$. In one experiment a gram was exposed to the laboratory atmosphere for 36 days, during which the relative humidity probably averaged about 30 percent; the sample was reweighed at various intervals, 7 times in all, and no change in the weight could be detected. In a second experiment a $0.4-\mathrm{g}$ sample showed no gain in weight during a total exposure of 7 days to about 60-percent relative humidity at $25^{\circ} \mathrm{C}$, or during a subsequent 8 days' exposure to about 80 -percent relative humidity. ${ }^{10}$ These experiments do not support Hoeflake's assertion [18] that anhydrous diphenyl phosphate takes up two molecules of water in the air. It was not possible to repeat the experiments by which Hoeflake reached her conclusion, as they were not described. Our experiments indicate that no difficulty should be experienced in maintaining diphenyl phosphate as anhydrous crystals under usual laboratory conditions.

However, when in the fused condition anhydrous diphenyl phosphate was found to gain weight temporarily in a moist atmosphere. About a gram of the acid was placed in a porcelain boat in an Abderhalden drying apparatus which was open to the air, and the sample was heated to fusion by

10 Approximately 60-percent relative humidity was achieved by keeping the sample in a desiccator containing saturated aqueous sodium bromide in contact with sodium bromide dihydrate. For 80-percent relative humidity, saturated aqueous ammonium chloride in contact with the solid salt was used allowing ethyl acetate to reflux in the apparatus. Over a period of 9 days the sample was repeatedly heated, cooled, and reweighed. During this time the weight fluctuated; the greatest gain, which occurred during rainy weather, was 2.2 percent. Water was then placed in the absorption bulb of the Abderhalden, the bulb was attached to the apparatus with partial evacuation, and the sample was remelted. In 3 days it gained more than 10 percent in weight; for complete conversion to the dihydrate a 14.4-percent gain in weight would have been necessary. An accurate measurement of the total gain was impossible as the sample rapidly lost weight while on the balance; the loss became less rapid after the weight had dropped to about a 5 -percent increase over the original weight. During 3-days' storage in the Abderhalden apparatus over phosphorus pentoxide at room temperature the sample became crystalline once more and regained its initial weight.

\section{b. Diphenyl Phosphate Dihydrate}

Some anhydrous diphenyl phosphate was dissolved in hot water and, after cooling in a refrigerator, the solution was seeded. Leaflets immediately started to form. After filiration these were partly dried between filter papers, then kept in a desiccator at 60-percent relative humidity (see footnote 10). After 12 days at $25^{\circ} \mathrm{C}$, during which the weight of the crystals decreased about 1.6 percent, the molecular weight found by titration corresponded closely to the theoretical molecular weight of the dihydrate $(286.2)$. The same result, within the limits of error, was obtained after further exposure of the material to 60-percent relative humidity for 25 days. On the assumption of an inert impurity (most likely, occluded water) the titrations indicated the dihydrate to be approximately 99.8 percent pure. A sample quickly liquefied when the tube containing it was placed in the meltingpoint bath at $49^{\circ}$ to $50^{\circ} \mathrm{C}$; bubbles formed, but they soon disappeared, leaving a clear, colorless liquid. However, the dihydrate did not melt sharply when heated gradually from a lower temperature. For example, a sample placed in the bath at $40^{\circ}$ showed noticeable softening at about $41.5^{\circ}$ and gradually liquefied, mostly at about $49^{\circ}$ to $50^{\circ}$.

As mentioned in section 2.2.a, a sample of fused, anhydrous diphenyl phosphate that had gained weight in a moist atmosphere returned to its original weight within 3 days when stored over phosphorus pentoxide. Slightly moist crystals of the dihydrate (about $2 \mathrm{~g}$ ) likewise lost weight when stored in a desiccator over anhydrous calcium sulfate. Within 100 days, while kept at a temperature that was automatically maintained close to $25^{\circ} \mathrm{C}$, the sample lost 13.2 percent in weight; if the sample had been completely air-dry the loss during conversion to the anhydrous acid should have been 12.6 percent. The molecular weight (by titration) of the dried acid was the same as that of anhydrous diphenyl phosphate, within the limits of error. 
Further study of the interconversion of anhydrous and dihydrated diphenyl phosphate would be of interest. However, the evidence seems sufficient to justify the conclusion that the anhydrous form of the acid can be maintained at constant composition more conveniently and reliably than the dihydrated form.

\subsection{Solubilities in Various Solvents}

In studies being made at the Bureau it is useful to know the approximate solubilities of anhydrous diphenyl phosphate in benzene, cyclohexane, and water. The results of some measurements are summarized in table 1 . Other available information

TABLE 1. Approximate solubilities of diphenyl phosphate in various solvents at $25^{\circ} \mathrm{C}$

\begin{tabular}{|c|c|}
\hline Solvent & $\begin{array}{c}\mathrm{g} \text { of solute per } 100 \mathrm{~g} \\
\text { of solvent }\end{array}$ \\
\hline $\begin{array}{l}\text { Acetone } \\
\text { Benzene } \\
\text { Carbon tetrachloride } \\
\text { Cyclohexane } \\
\text { Ether } \\
\text { Water....... }\end{array}$ & $\begin{array}{l}100 \text { a } \\
170 \text { b c } \\
4 \text { a } \\
1.5 \text { b d e } \\
100 \text { a } \\
3 \text { a b d }\end{array}$ \\
\hline
\end{tabular}

a This value, given in [2], was evidently obtained using hydrated diphenyl phosphate.

b Determined in this laboratory by a titration procedure, using anhydrous diphenyl phosphate.

The value given in [2] is 10 ; see footnote

d The same value was obtained by weighing the residue left after evaporation of solvent.

e Infrared measurements by James Stewart of the Bureau indicated a benzene content less than 0.01 percent.

on solubilities is included in the table. Early investigators reported that diphenyl phosphate is easily soluble in alcohol, benzene, chloroform, and ether [14 to 17].

The solubility of diphenyl phosphate in benzene is difficult to measure because supersaturation tends to occur and a slight change in the temperature has a marked effect. However, good agreement was obtained in duplicate experiments. Partial hydration of the sample used may account for the much lower solubility reported in [2].

The earliest investigators [14 to 16$]$ stated that diphenyl phosphate is practically insoluble in water, but later [17] 1 part of the acid was reported to be soluble in 35 parts of water. This value is close to the one measured at the Bureau and also reported in [2]. Crystals of anhydrous diphenyl phosphate liquefy as soon as they are brought in contact with a little water. On shaking, the liquid separates into minute, scarcely visible drops. As a result of this behavior, in some early aqueous titrations of diphenyl phosphate incomplete solution of the samples occurred without detection, even when the quantity used was much below the amount required for a saturated solution. It proved advantageous to dissolve the crystals in a little ethanol, then dilute to the desired volume with water.

\subsection{Acidic Strength}

Some years ago Morton [22] concluded from comparative potentiometric titrations of orthophos- phoric acid and monophenyl phosphate in water at $18^{\circ} \mathrm{C}$. that replacing one of the hydrogen atoms of orthophosphoric acid by a phenyl group increases the acidity. Subsequently, Kumler and Eiler [23] measured the relative acidic strengths of four monoalkyl esters (methyl, ethyl, $n$-propyl, and $n$-butyl) and the corresponding dialkyl esters of orthophosphoric acid in water at $25^{\circ} \mathrm{C}$. Their data showed that the monoalkyl esters are stronger acids than phosphoric acid, and that the dialkyl esters are stronger still. Morton's data indicate that monophenyl phosphate is stronger than the monoalkyl phosphates investigated by Kumler and Eiler, although the experimental conditions used in the two sets of investigations were too different to permit a numerical comparison.

It was therefore to be expected that diphenyl phosphate would show strongly acidic behavior in water, and this proved to be the case. In fact, the curves obtained from potentiometric titrations with $0.1 \mathrm{~N}$ alkali in water were practically identical with curves obtained for hydrochloric acid of equivalent strength. Moreover, the measured $p \mathrm{H}$ values of several aqueous solutions ${ }^{11}$ of diphenyl phosphate having concentrations in the range from about 0.005 $M$ to $0.1 M$ were in very good agreement with the $p \mathrm{H}$ values calculated on the assumption of complete ionization.

As will be shown in section 4, diphenyl phosphate also behaves as a strong acid in benzene, and appears to be stronger in methanol than in water. However, in acetic acid it behaves like a rather weak acid.

\subsection{Stability in Aqueous and Alcoholic Solutions}

Experiments to be described in section 4 showed that diphenyl phosphate can be titrated with bases very successfully in benzene, methanol, water, and mixtures of these solvents, and therefore merits attention as a possible acidimetric standard in some or all of these solvents. However, if it underwent rapid chemical change in solution, such as hydrolysis or alcoholysis, its usefulness would be limited.

The literature contains fragmentary and partially conflicting statements about the stability of diphenyl phosphate and closely related esters in aqueous and alcoholic solutions. Glutz [14] stated that only one phenyl group is split off when triphenyl phosphate is treated with strong potash, and Autenrieth [17] reported the same result from heating various triaryl phosphates with an equivalent of alcoholic potash. As further evidence of the stability of diaryl phosphates, Autenrieth claimed that they are not much saponified in dilute aqueous acidic or alkaline solutions, even on long heating.

However, Plimmer and Burch [24] concluded that all three phenyl esters of orthophosphoric acid are completely hydrolyzed on heating with acid or alkali to $85^{\circ}$ for periods as long as a week; 5 -percent solutions of the esters in $1 N$ hydrochloric acid or sodium hydroxide were used, and at appropriate intervals analyses for phenol and inorganic phosphate were made. The comparative behavior of some alkyl

11 Each solution contained about 10 percent of ethanol by volume. 
phosphates was also studied. The general conclusions were as follows: (1) All three phenyl esters of orthophosphoric acid can be completely hydrolyzed by dilute alkali, though a trialkyl phosphate is not hydrolyzed beyond the dialkyl ester; (2) all phenyl and alkyl esters can be completely hydrolyzed in dilute acid. Plimmer and Burch also mentioned that at $40^{\circ}$ diphenyl phosphate was appreciably hydrolyzed by alkali in 24 hours.

Smith and Stohlman [25] found that 1-percent triphenyl phosphate in half-saturated aqueous sodium hydroxide is completely hydrolyzed to phenol and inorganic phosphate after about 5 hours' refluxing; the three isomeric tricresyl phosphates behaved analogously, except that they seemed to be more resistant to hydrolysis. In absolute ethanol tri-ocresyl phosphate was hydrolyzed to di-o-cresyl phosphate at $25^{\circ} \mathrm{C}$ to an extent that was proportional to the concentration of potassium hydroxide present, but no removal of $o$-cresol from either di-o-cresyl phosphate or mono-o-cresyl phosphate was detected, even when the concentration of alkali was as high as $2.5 N$ and the experiment lasted 9 days.

The conclusions of Breusch and Keskin [26] are partially in conflict with those of Plimmer and Burch. Their results from refluxing various triaryl phosphates $(0.01 M)$ in aqueous sulfuric acid or sodium hydroxide, with periodic analysis of small samples for inorganic phosphate, may be summarized as follows: (1) Triaryl phosphates are easily saponified in hot $1 N$ alkali, but are stable toward sulfuric acid (1 to $5 \mathrm{~N}$ ), even on heating for hours; (2) tribenzyl phosphate is saponified by both acid and alkali. In explanation of the results, the authors suggested that tribenzyl phosphate is a true ester, but triaryl phosphates are rather to be regarded as acid anhydrides.

Enzymatic hydrolysis of diphenyl phosphate and other esters of phosphoric acid has been studied, mostly in Japan; the extent of hydrolysis reportedly varies with the nature of the phosphatase and the substrate and also with the $p \mathrm{H}$. An interesting study has been made of the mechanism of hydrolysis of trimethyl phosphate in alkaline, acidic, and initially neutral aqueous solutions, with the use of water containing an excess of $\mathrm{O}^{18}[27]$. The data indicate that in alkaline solution hydrolysis proceeds entirely through rupture of the $\mathrm{P}-\mathrm{O}$ linkage, whereas in acid solution rupture involves mainly, if not exclusively, the $\mathrm{O}-\mathrm{CH}_{3}$ bond.

While recrystallizing diphenyl phosphate from water we observed that protracted boiling of an aqueous solution produces a slight milkiness and an odor of phenol. On the other hand, no change in the acidity of an essentially aqueous (see footnote 11) $0.1 M$ solution was observed during 156 days' storage at $25^{\circ} \mathrm{C}$ (see table 2). It seems clear that, while high temperatures must be avoided, diphenyl phosphate is sufficiently stable in neutral aqueous or alcoholic solutions to warrant further investigations of its utility as an acidimetric standard at room temperature. More rigorous tests, including intercomparisons with other acidimetric standards, will of course be necessary before it can be recommended for highly accurate measurements.
TABLE 2. Stability of $0.1 M$ diphenyl phosphate in water at $25^{\circ} C^{\text {a }}$

\begin{tabular}{|c|l|}
\hline $\begin{array}{c}\text { Time elapsed since } \\
\text { preparation }\end{array}$ & $\begin{array}{c}\text { Molarity by titration } \\
\text { with } 0.1 ~ N \text { alkali }\end{array}$ \\
\hline Days & \\
0 & \\
12 & $0.0987,0.0989$. \\
98 & $0.0985,0.0986,0.0985$ \\
156 & $0.0989,0.0989$ \\
& $0.0988,0.0988$. \\
\hline
\end{tabular}

a The acid was dissolved with the aid of $20 \mathrm{ml}$ of 95 -percent ethanol, the final volume being $200 \mathrm{ml}$

\section{Use of Diphenyl Phosphate in Acid-Base Titrations in Various Aqueous and Non- aqueous Solvents}

\subsection{Titrations of Diphenyl Phosphate With Sodium Hydroxide in Water}

The results of some titrations of two different lots of purified diphenyl phosphate are presented in table $3 .^{12}$ According to the results shown in the table, both lots of diphenyl phosphate were approximately 99.9 percent pure.

TABLE 3. Titrations of diphenyl phosphate in water a

\begin{tabular}{|c|c|c|c|}
\hline \multirow{2}{*}{$\begin{array}{l}\text { Weight of } \\
\text { sample }\end{array}$} & \multicolumn{2}{|c|}{$\mathrm{NaOH} b$} & \multirow[b]{2}{*}{ Purity } \\
\hline & $\begin{array}{l}\text { Calcu- } \\
\text { lated }\end{array}$ & Found & \\
\hline \multicolumn{4}{|c|}{ Titrations using phenolphthalein } \\
\hline $\begin{array}{c}g \\
\text { c } 1.0025 \\
\text { c } 0.9898 \\
\text { с } 1.0437 \\
\text { с } 1.0510 \\
\text { c } 1.0966 \\
\text { c } 1.0307 \\
\text { с } 1.0193\end{array}$ & $\begin{array}{c}m l \\
35.68 \\
35.23 \\
37.15 \\
37.41 \\
39.03 \\
36.68 \\
36.28\end{array}$ & $\begin{array}{c}m l \\
35.69 \\
35.18 \\
37.12 \\
37.36 \\
39.00 \\
36.59 \\
36.23\end{array}$ & $\begin{array}{r}\% \\
100.03 \\
99.86 \\
99.92 \\
99.87 \\
99.92 \\
99.75 \\
99.86\end{array}$ \\
\hline Average $^{\circ}$ & $-\cdots$ & $-\ldots$ & 99.89 \\
\hline \multicolumn{4}{|c|}{ Potentiometric titrations } \\
\hline $\begin{array}{r}\text { c } 0.3576 \\
\text { c. } 3222 \\
\text { d. } 3962 \\
\text { d. } 4213 \\
\text { d. } 4838\end{array}$ & $\begin{array}{l}12.75 \\
11.49 \\
14.13 \\
15.02 \\
17.25\end{array}$ & $\begin{array}{l}12.74 \\
11.47 \\
14.15 \\
15.01 \\
17.24\end{array}$ & $\begin{array}{r}99.92 \\
99.83 \\
100.14 \\
99.93 \\
99.94\end{array}$ \\
\hline
\end{tabular}

a In titrations with phenolphthalein, the samples were dissolved in $15 \mathrm{ml}$ of 95-percent ethanol and $85 \mathrm{ml}$ of water; in the potentiometric titrations, the re. s5-percent ethanol amounts of ethanol and water were 10 and $90 \mathrm{ml}$

spective amounts of ethanol and water were 10 and $90 \mathrm{ml}$.
b The normality (about $0.1 \mathrm{~N}$ ) was determined by standardization against b The normality (about $0.1 \mathrm{~N}$ ) was determined b
NBS Standard Sample of potassium acid phthalate.

$\mathrm{c}, \mathrm{d}$ The superseripts $\mathrm{c}$ and $\mathrm{d}$ indicate two different lots of purified diphenyl phosphate.

\subsection{Titrations of Bases With Diphenyl Phosphate in Glacial Acetic Ácid}

At the present time 70-percent perchloric acid is the only generally satisfactory reagent for preparing standard acid solutions for titrations of bases in glacial acetic acid. Acetic anhydride must be added to destroy water introduced with the reagent, and the concentration of the solution must always be deter-

12 See also table 2 and section 4.3. As was pointed out in section 3.3 it is advisable to add a little alcohol to ensure complete solution of samples of diphenyl phosphate used in aqueous titrations. 
mined by titration against a primary basic standard, such as diphenylguanidine, potassium acid phthalate, or sodium carbonate. Clearly, it would be very gratifying to find a solid, anhydrous acid, comparable in strength with perchloric acid. Diphenyl phosphate would not be expected to equal perchloric acid in strength, but it was desirable to ascertain the comparative behavior of the two acids in glacial acetic acid.

The titration curves presented in figure 2 show that diphenyl phosphate was not satisfactory as a replacement for perchloric acid in the potentiometric titration of di-o-tolylguanidine and guanidine acetate in glacial acetic acid. Curves obtained in the titration of potassium acid phthalate and tribenzylamine with diphenyl phosphate were very similar to those shown. ${ }^{13}$ There is a recognizable break in all of the titration curves in the expected region $(8.4 \mathrm{ml})$, but in the titrations with diphenyl phosphate the break is not steep enough for any but crude analy tical procedures. ${ }^{14}$ Dyes (for example, crystal violet) which are commonly used as indicators for titrations in glacial acetic acid change color in the emf region -450 to -550 $\mathrm{mv}$, and plainly would be completely useless in titrations with diphenyl phosphate.

In a few miscellaneous titrations of sodium acetate with diphenyl phosphate in glacial acetic acid mixed with other solvents-benzene, dioxane, ethanol, water-the results were much the same. In general, the microvoltmeter readings were very steady except for mixtures containing a high proportion of benzene. ${ }^{15}$

13 The two bases referred to in figure 2 did not give a precipitate with either diphenyl phosphate or perchloric acid; the perchlorates, but not diphenyl phosphates, produced upon titrating potassium acid phthalate and tribenzylamine precipitate under the conditions used.

14 It should be noted that the titrations were only of an exploratory character, and the results with diphenyl phosphate would probably have been a little better if the solutions had been completely anhydrous. The solutions were better if the solutions had been completely an

estimated to contain about 0.6 percent of water. hydrogen bonding. No one seems to have made any studies of aryl phosphates hydrogen bonding. No one seems to have made any studies of aryl phosphates
which throw any direct light on their tendency to undergo self-association or which throw any direct light on their tendency to undergo self-association or
otherwise participate in hydrogen bonding, but tributyl phosphate has been otherwise participate in hydrogen bonding, but tributyl phosphate has been
found useful for extracting acetic acid from aqueous mixtures with hydrochloric acid [28], and several other studies indicate that oxygen attached to phosphorus is sometimes a very effective electron donor and that some acids of phosphorus show a tendency to form aggregates in benzene [1(a) (p. 257), 29 to 32].

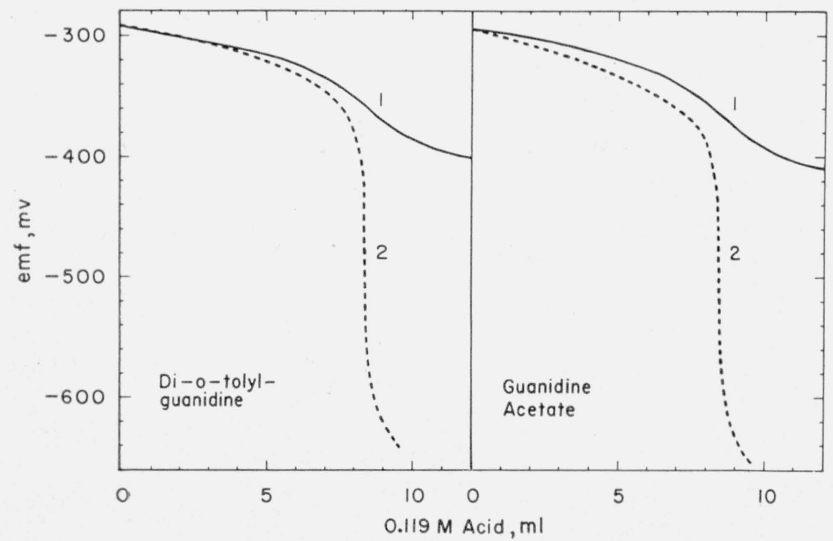

Figure 2. Comparative behavior of (1) diphenyl phosphate and (2) perchloric acid in the potentiometric titration of di-otolylguanidine and of guanidine acetate in glacial acetic acid.

Electrodes: glass, saturated calomel. Initial solutions contained 0.001 mole of the base in $30 \mathrm{ml}$.

\subsection{Comparative Titrations of Diphenyl Phosphate and Carboxylic Acids With Sodium Methylate in Solvents of Various Compositions}

\section{a. Potentiometric Titrations}

In preliminary potentiometric titrations of 5- or $10-\mathrm{ml}$ portions of $0.1-M$ diphenyl phosphate with sodium methylate (approx $0.115-M$ ) in methanol, the precision was very good (usually $\pm 0.01 \mathrm{ml}$ ), even though the temperature of the laboratory was subject to fluctuations (mostly in the range $25^{\circ} \pm 2^{\circ} \mathrm{C}$ ) and methanol has a rather high coefficient of thermal expansivity. Carboxylic acids, of which acetic, benzoic, and palmitic acids are typical, are often dealt with in nonaqueous titrations. Solutions of these three acids were also titrated with sodium methylate in methanol, under conditions paralleling those used in titrations of diphenyl phosphate. The titration curves indicated that diphenyl phosphate is a much stronger acid than the three carboxylic acids, in harmony with the evidence that diphenyl phosphate is a strong acid in water.

The ready solubility of both diphenyl phosphate and benzoic acid in benzene, methanol, and water suggested the possibility of observing what alterations in the titration curves would result from changing the solvent gradually from methanol toward benzene, on the one hand, and toward water, on the other. For this, 5-ml samples of $0.1 M$ acid in methanol were diluted to approximately $50 \mathrm{ml}$ with various proportions of methanol and benzene or methanol and water. The samples were then titrated with sodium methylate, dissolved in a solvent of approximately the same composition as that of the solution being titrated. The results are shown in figure $3 .^{16}$

It is evident from the figure that for an aqueous solution of either acid a negative value on the emf scale would correspond to a low $p \mathrm{H}$ value, and an increasingly positive emf value would indicate a gradual increase in the $p \mathrm{H}$ of the solution. In the case of diphenyl phosphate, the initial acidityas registered by the emf reading - was decidedly reduced when the solvent was changed from nearly pure methanol to predominantly water. Hardly any initial effect on the acidity was observable on changing from methanol to $17: 3$ benzene-methanol. In the case of benzoic acid, changing the solvent from nearly pure methanol to $9: 1$ water-methanol again produced a striking effect on the initial acidity, this time a decided increase in the acidity. As with diphenyl phosphate, comparatively little change in the initial acidity resulted from changing from methanol to $17: 3$ benzene-methanol.

16 The titrant was prepared by diluting $1.15 \mathrm{M}$ sodium methylate in methanol to $0.115 \mathrm{M}$ in a volumetric flask, first adding any methanol and then diluting to to $0.115 \mathrm{M}$ in a volumetric flask, first adding any methanol and then diluting to volume with either benzene or water. The samples of 1 . $M$ acid in metric the added were transferred to the titration beakers by means of a volumetric pipet; the added solvents, totalling $45 \mathrm{ml}$ in volume before mixing, were measured in graduated
cylinders. The solvents containing the sample and the titrant were thus similar, cylinders. The solvents containing the sample and the titrant were thus similar, although not identical, in composition. A little water was, of course, introduced into each solution with the methanol, and a minute quantity could also enter The quantities of water thus introduced were adequate for proper functioning of the glass electrode. The solutions that yielded curves 5 had such a high water content as to be practically the same as aqueous solutions. 


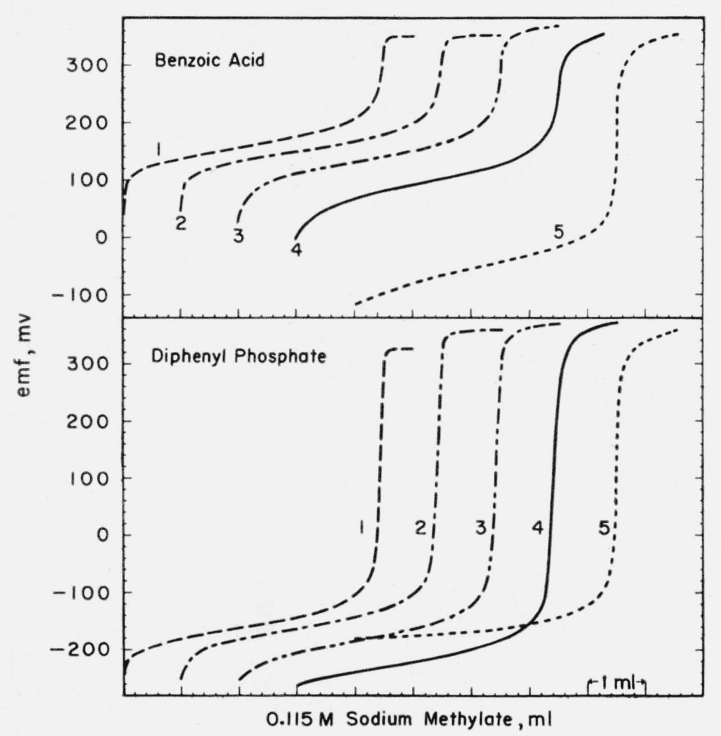

Figure 3. Effect of composition of the solvent in the potentiometric titration of benzoic acid and of diphenyl phosphate with sodium methylate.

Electrodes: glass, saturated calomel. Initial solution: $50 \mathrm{ml}$ of $0.01-M$ acid. Composition of solvent: (1) benzene-methanol, 17:3 (by volume); (2) benzenemethanol, $4: 1$; (3) benzene-methanol, $1: 1$; (4) methanol containing 0.5 weight percent of water; (5) water-methanol, $9: 1$. Curves 2 to 5 have each been shifted $1 \mathrm{ml}$ to the right of the preceding curve.

Figure 4 shows the results of titrating a mixture of equivalent quantities of diphenyl phosphate and benzoic acid in three different solvents. ${ }^{17}$ The buffering effect of benzoic acid on the end-point for the titration of diphenyl phosphate in water (curve 3 ) was absent in the titrations in methanol or benzene-methanol, thus making possible a sharp determination of the end-point for each acid. The results support Hantzsch's assertion that water "levels" the strengths of acids, making strong acids weaker and weak acids stronger [33]. An "unleveling" of the strengths of two such acids as benzoic acid and diphenyl phosphate can be brought about by changing to a nonaqueous solvent like methanol or benzenemethanol. While benzoic acid is thereby reduced in apparent strength, the reduction is not sufficient to impair seriously the accuracy of the titration. A few examples of a related character have been reported previously by others $[34,35]$.

\section{b. Titrations in 4:1 Benzene-Methanol Using Indicators}

Benzene-methanol, 4:1 by volume, is currently popular as a solvent for nonaqueous titrations of carboxylic acids [36], particularly if the acid is one of bigh molecular weight. As was shown in figure 4 (curve 1), when an equimolar mixture of benzoic acid and diphenyl phosphate in this solvent was titrated potentiometrically with sodium methylate, the separate end-points could be located satisfactorily. In practice, it would often be more convenient to locate both such end-points by the use of indicators. As a preliminary step in finding a suitable pair of

17 Note that distances along the vertical scale are twice as great as in figure 3 .

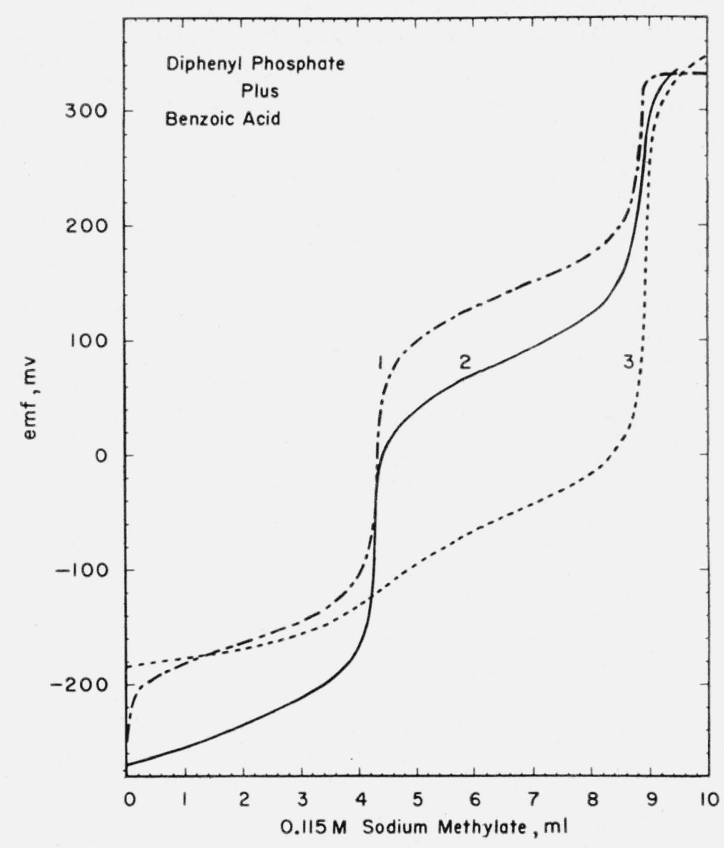

Figure 4. Effect of composition of the solvent in the potentiometric titration of a mixture of diphenyl phosphate and benzoic acid with sodium methylate.

Electrodes: glass, saturated calomel. Initial volume: $50 \mathrm{ml}$. Initial concentration of each acid: $0.01-M$. Composition of solvent: (1) benzene-methanol, 4:1 (by volume); (2) methanol containing 0.5 weight-percent of water; (3) watermethanol, $9: 1$.

indicators, 5-ml samples of $0.1-M$ diphenyl phosphate were repeatedly titrated potentiometrically with sodium methylate, each time with the addition of a different indicator dye, and the approximate emfs at which the color changes of the various indicators occurred were noted. For the purposes of the experiment, it was satisfactory to regard the indicator end-point as the emf midway between the emf values obtained immediately before and after addition of the drop of alkali that produced the most striking color change. ${ }^{18}$ The results thus obtained with 17 different indicator dyes are shown in figure 5. It will be noted that three of the symbols are enclosed in parentheses $(\alpha-\mathrm{NR}, p-\mathrm{EC}, \mathrm{MR})$; the parentheses are used here to signify that these basic azo dyes did not undergo a sharp color change at the end-point. For all of the other indicators, the color change at the end-point was very sharp. In the cases of the sulfonephthaleins (BPB, BCG, BCP, PR, BTB, CR, CP, TB) and phenolphthalein, there was a roughly linear relationship between the emf values in $4: 1$ benzene-methanol at our arbitrarily set end-points and the mid-points of the $p \mathrm{H}$ ranges as commonly given for aqueous solutions of these indicators. Nile blue $\mathrm{A}$ and Nile blue $2 \mathrm{~B}$ did not fit into this linear relationship - their color change in benzene-methanol occurred in too negative an emf region.

${ }^{18}$ It should be made clear that the end-points thus arbitrarily established are only approximate. For the most part, only one titration of a kind was performed. Indicators were dissolved in methanol, benzene, or benzene-methanol; the con: Indicators were dissolved in methanol, benzene, or benzene-methanol; the conThe amounts added ( 1 to 5 drops) were such that one drop of sodium methylate produced a vivid color change at the end-point. 


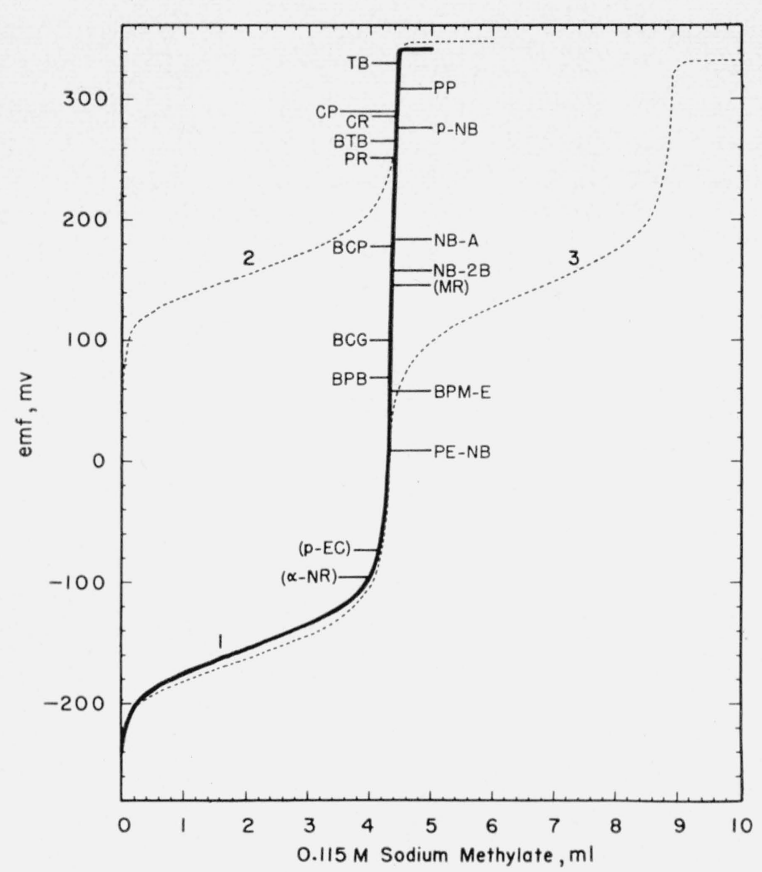

Figure 5. Titration of (1) diphenyl phosphate, (2) benzoic acid, and (3) a mixture of diphenyl phosphate and benzoic acid with sodium methylate in 4:1 benzene-methanol.

Initial volume, $50 \mathrm{ml}$; initial concentration of each acid, $0.01 \mathrm{M}$. Electrodes: glass, saturated calomel. Indicators: $\alpha$-NR, $\alpha$-naphthyl red; $p$-EC, $p$-ethoxy chrysoidin; PE-NB, phenyl diethyl Nile blue; BPM-E, bromophthalein magent E; B PB, bromophenol blue; BCG, bromocresol green; MR, methyl red; NB-2B Nile blue 2B; BCP, bromocresol purple; NB-A, Nile blue A; PR, phenol red; BTB, bromothymol blue; $p$-NB, $p$-naphtholbenzein; CR, 0 -cresol red; CP, $m$-cresol purple; PP, phenolphthalein; TB, thymol blue.

It is clear from figure 5 that all of the indicators tried, except $\alpha$-naphthyl red, $p$-ethoxychrysoidin, and methyl red, should be usable for titrating diphenyl phosphate under the conditions chosen. Bromophthalein magenta $\mathrm{E}$ is one of the best. $p$-Naphtholbenzein and the three Nile blues are also excellent. The relative vertical position of the Nile blues is that which would be predicted from the differences in structure (see section 2.4.c). Some of the sulfonephthaleins (PR, CR, CP, TB) showed a dual color change, as in water; however, the color change in the acid region was very gradual. Other sulfonephthaleins (BPB, BCG, BCP, BTB) were practically colorless at first, then gradually became a bright yellow as sodium methylate was added, eventually undergoing the characteristic vivid color change at the end-point. ${ }^{19}$

Two additional curves (lightly dashed) are shown in figure 5-the titration curve for benzoic acid alone and the titration curve for an equimolar mixture of diphenyl phosphate and benzoic acid. For the mixture of the two acids, as well as for benzoic acid alone, the choice of indicators is greatly narrowed. Phenyl diethyl Nile blue is the only possible indicator in the group studied which can be used for the first end-point. Bromophthalein

\footnotetext{
19 Analogous changes in color of these dyes in benzene were reported earlier,
} and the probable structural changes involved were discussed [37]. magenta $\mathrm{E}$ changes color just a little too late (and therefore too gradually). The 5 topmost indicators should be usable for the second end-point. The one that we liked best was $p$-naphtholbenzein, the indicator that was adopted for an important ASTM procedure for determining acidity in petroleum products dissolved in benzene-isopropanol [38]. The combination of phenyl diethyl Nile blue and $p$ naphtholbenzein was very satisfactory for titrating the mixture of diphenyl phosphate and benzoic acid; the first color change was greenish blue to violet pink and the second color change, salmon pink to violet. It is preferable to add $p$-naphtholbenzein after reaching the first end-point; both indicators can be added at the start, but the first end-point is then a little less striking. The same combination of indicators should, of course, be usable for other pairs of acids comparable in strength to diphenyl phosphate and benzoic acid, and the method used for selecting suitable indicators is of still wider application.

\subsection{Comparative Titrations of Diphenylguanidine, Triethylamine, and Tris(hydroxymethyl)amino- methane With Diphenyl Phosphate in Solvents of Various Compositions}

1,3-Diphenylguanidine, triethylamine, and tris(hydroxymethyl)aminomethane were titrated with diphenyl phosphate in the same series of solvents as those listed under figure 3 and also in 1:1 watermethanol. The titration curves are shown in figure 6 . The conditions were closely similar to the ones used in the titrations of diphenyl phosphate and benzoic acid..$^{20}$ The values obtained in titrating triethylamine were a little low and not closely reproducible, probably indicating some volatilization of the amine; the loss seemed to be least in the solutions containing the highest proportion of methanol.

These bases were selected as representing a large group of organic bases having aqueous dissociation constants, expressed as $p K_{b}$, in the approximate range 3 to $6 .^{21}$ The tendency for triethylamine to volatilize permits only a tentative comparison of its behavior with that of the other two bases. However, the curves suggest that triethylamine is more affected in strength than the other two bases when the water content of the solvent is progressively reduced and the benzene content is increased. There are also indications that diphenylguanidine is stronger than triethylamine in methanol and benzene, although weaker in water. Such a conclusion would be in harmony with results obtained in spectrophotometric studies of the comparative reactivities of triethylamine and diphenylguanidine with bromophthalein magenta $\mathrm{E}$ in benzene [1,39].

${ }^{20}$ In the titration of diphenylguanidine a solution $0.01-M$ in $9: 1$ water-methanol could not be prepared. The sample was dissolved in $100 \mathrm{ml}$ of solvent (instead of the usual $50 \mathrm{ml}$ ), with the aid of about $4 \mathrm{ml}$ of the standard diphenyl phosphate

solution, and the titration was then continued from that point (curve 6).
21 Values of $p K_{b}$ for triethylamine, diphenylguanidine, and tris(hydroxymethyl)aminomethane are $3.3,4.0$, and 5.9 , respectively $[1,4]$. 


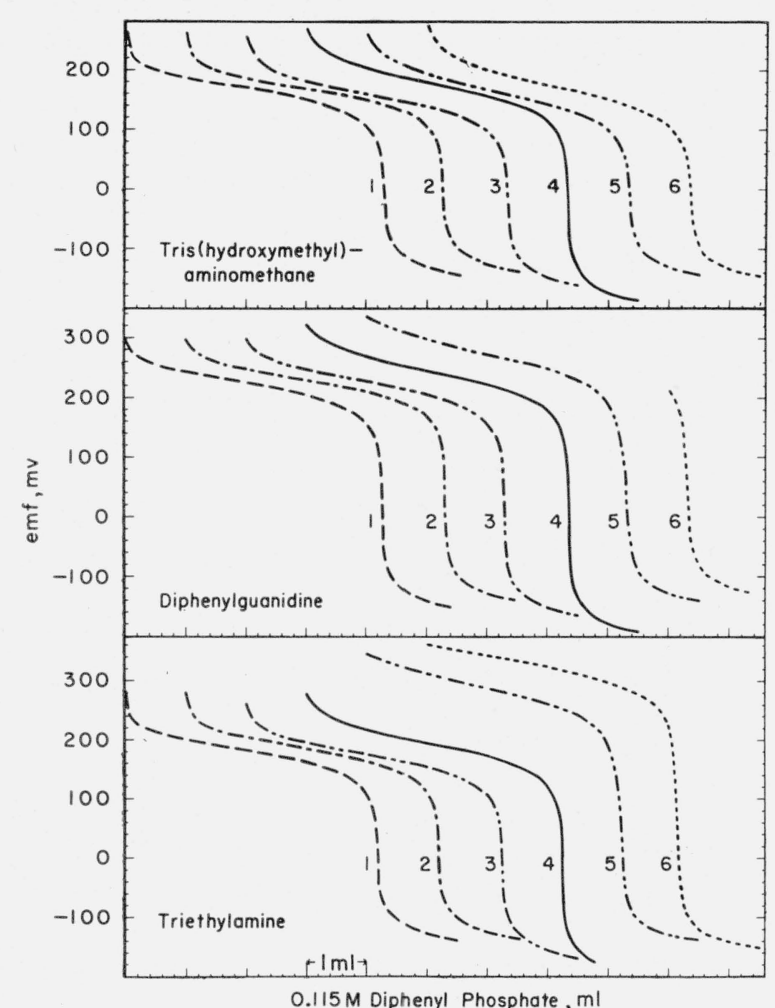

Figure 6. Effect of composition of the solvent in the potentiometric titration of tris(hydroxymethyl)aminomethane, 1,3diphenylguanidine, and triethylamine with diphenyl phosphate

Electrodes: glass, saturated calomel. Initial solution: $50 \mathrm{ml}$ of $0.01-M$ base (for exceptions, see text). Composition of solvent: (1) benzene-methanol, 17:3 (by volume); (2) benzene-methanol, 4:1; (3) benzene-methanol, 1:1; (4) methanol containing 0.5 weight-percent of water; (5) water-methanol, $1: 1$; (6) water-methanol, $9: 1$. Curves 2 to 6 have each been shifted $1 \mathrm{ml}$ to the right of the preceding curve.

\subsection{Titrations of Diphenylguanidine and Di-o-tolyl- guanidine With Diphenyl Phosphate in Benzene, Using Indicators}

\section{a. General Discussion}

Acid-base titrations using only benzene as the solvent differ from the titrations discussed in preceding sections in that ions play a very minor role. The reaction in benzene or other aprotic solvents consists essentially of the combination of the base with the acid to form a salt. An illustration is the combination of an amine with a monobasic, nonassociated acid, as follows:

$$
\begin{aligned}
& \mathrm{R}_{3} \mathrm{~N}+\mathrm{HA} \rightleftarrows \mathrm{R}_{3} \mathrm{NH}^{+} \ldots \mathrm{A}^{-} . \\
& \text {(amine) (acid) (salt) }
\end{aligned}
$$

As indicated in the equation, the major part of the salt is not separated into ions when the solvent is benzene or a like solvent; instead, the salt behaves more like a highly polar molecule. ${ }^{22}$ The end-point

${ }^{22}$ It should be noted, however, that in spite of the minute concentration of free ions in such a solution, LaMer and Downes successfully performed certain acid-base titrations potentiometrically and conductimetrically in benzene [40], and Maryott performed some additional acid-base titrations conductimetrically in benzene [4I]. can easily be located by adding a minute amount of an indicator dye, which likewise forms a salt according: to eq (1). The dye can, of course, be either an amine or an acid, but it must undergo a striking change in color when converted to a salt.

Vorländer [42] was the first to perform titrations involving the chemical reaction shown as eq (1); he referred to this type of titration as "aminometry" or "alkalimetry without hydroxyl ions." ${ }^{23}$ In Europe and Australia aminometry has been applied successfully to a large variety of organic bases, and the method would probably find more widespread application if acids of suitable strength and purity were readily obtainable. ${ }^{24}$

\section{b. Titrations of Diphenylguanidine and Di-o-tolylguanidine With Diphenyl Phosphate in Benzene}

Few solid organic bases of strengths comparable with those of the aliphatic amines $\left(p K_{b} \cong 3\right.$ to 5$)$ are readily available. Important among the few are 1,3-diphenylguanidine, $\mathrm{C}_{6} \mathrm{H}_{5} \mathrm{NH}(\mathrm{C}=\mathrm{NH}) \mathrm{NHC}_{6} \mathrm{H}_{5}$, and the closely related compound, 1,3-di-o-tolylguanidine. Previously benzene solutions of these bases have been titrated satisfactorily with $d$-camphorsulfonic acid, picric acid, and trichloroacetic acid in this laboratory [1]. As shown in tables 4 and 5, the two bases can also be titrated successfully with diphenyl phosphate in benzene.

Table 4 presents two titrations of $0.05-M$ diphenylguanidine with $0.05-M$ diphenyl phosphate with bromophthalein magenta $\mathrm{E}$ as the indicator. In an acid solution the indicator is yellow; the color in a basic medium is magenta to blue, depending on the concentration and on the solvent [1, 39]. Experiments 1 and 2 were performed under slightly different conditions, as indicated in the footnotes. In experiment 1 the solution gradually increased in its content of diphenylguanidinium diphenyl phosphate, becoming about $0.01-M$ at the end of the titration; the concentration of bromophthalein magenta $\mathrm{E}$ was about 60 percent as great at the end of the titration as at the beginning. Experiment 2 was performed in such a way as to maintain the content of diphenylguanidinium diphenyl phosphate approximately constant $(0.01-M)$ at each end-point; the concentration of bromophthalein magenta E was also the same at each end-point.

The change from yellow to magenta was always sharp. After this end-point had been reached, sufficient acid was added to restore the yellow color. This second end-point ought to be a little more accurate than the magenta end-point because to obtain the magenta color sufficient diphenylguanidine must be added to combine with both diphenyl phosphate and bromophthalein magenta E. The data do not indicate any advantage in performing

${ }^{23}$ A review of the literature and additional examples from work in this laboratory were given in [1].

${ }_{24}$ For successful titration the reaction (eq 1) must, of course, proceed to completion. Benzoic and acetic acids, for example, are much too weak to be used for such titrations. Vorländer used hydrogen chloride, dissolved in benzene, ligroin, or chloroform, and had to restandardize it daily. $p$-Toluenesulfonic acid is quite strong but the commercial monohydrate must be dehydrated by individual workers and is not of high purity. Picric acid has found some use, but is not as strong as would be desirable; other objectionable features are the yellow color of its salts and the hazards in handling the acid and its salts. 
TABLE 4. Titrations of 0.05- $M$ diphenylguanidine with 0.05-M diphenyl phosphate in benzene, using bromophthalein magenta $E$

\begin{tabular}{|c|c|c|c|c|c|}
\hline \multicolumn{2}{|c|}{ Acid } & \multirow{2}{*}{ Base } & \multirow{2}{*}{$\begin{array}{l}\text { Molarity } \\
\text { of salt }\end{array}$} & \multicolumn{2}{|c|}{$\begin{array}{l}\text { Strength of base } \\
\text { in terms of acid }\end{array}$} \\
\hline Yellow & Magenta & & & Yellow & Magenta \\
\hline \multicolumn{6}{|c|}{ Experiment $1^{\mathrm{a}}$} \\
\hline $\begin{array}{l}m l \\
1.11 \\
2.03 \\
3.03 \\
4.04 \\
5.06 \\
6.03 \\
7.07 \\
8.03 \\
9.02 \\
9.99\end{array}$ & $\begin{array}{c}m l \\
1.09 \\
2.01 \\
3.00 \\
4.01 \\
5.04 \\
6.01 \\
7.05 \\
8.00 \\
9.00 \\
9.96\end{array}$ & $\begin{array}{c}m l \\
1.08 \\
2.02 \\
3.01 \\
4.02 \\
5.04 \\
6.02 \\
7.06 \\
8.03 \\
9.02 \\
9.97\end{array}$ & $\begin{array}{l}0.0016 \\
.0029 \\
.0042 \\
.0053 \\
.0063 \\
.0071 \\
.008 \\
.0087 \\
.0094 \\
.01\end{array}$ & $\begin{array}{l}1.028 \\
1.00_{5} \\
1.007 \\
1.00_{5} \\
1.00_{4} \\
1.00_{2} \\
1.00_{1} \\
1.00_{0} \\
1.00_{0} \\
1.00_{2}\end{array}$ & $\begin{array}{r}1.00_{9} \\
.99_{5} \\
.99_{7} \\
.99_{8} \\
1.00_{0} \\
.99_{8} \\
.99_{9} \\
.99_{6} \\
.99_{8} \\
.99_{9}\end{array}$ \\
\hline A verage. & & & & $1.00_{5}$ & $.99_{9}$ \\
\hline \multicolumn{6}{|c|}{ Experiment $2^{\mathrm{b}}$} \\
\hline $\begin{array}{r}1.03 \\
2.02 \\
3.04 \\
4.04 \\
5.07 \\
6.03 \\
7.02 \\
8.03 \\
9.02 \\
10.00\end{array}$ & $\begin{array}{l}1.01 \\
2.00 \\
3.02 \\
4.02 \\
5.05 \\
6.01 \\
7.00 \\
8.00 \\
9.00 \\
9.98\end{array}$ & $\begin{array}{l}1.03 \\
2.02 \\
3.05 \\
4.06 \\
5.08 \\
6.03 \\
7.01 \\
8.03 \\
9.02 \\
9.99\end{array}$ & $\begin{array}{l}0.01 \\
.01 \\
.01 \\
.01 \\
.01 \\
.01 \\
.01 \\
.01 \\
.01 \\
.01\end{array}$ & $\begin{array}{r}1.00_{0} \\
1.00_{0} \\
.99_{7} \\
.995 \\
.99_{8} \\
1.00_{0} \\
1.00_{1} \\
1.00_{0} \\
1.00_{0} \\
1.00_{1}\end{array}$ & $\begin{array}{l}0.98_{1} \\
.99_{0} \\
.99_{0} \\
.99_{0} \\
.99_{4} \\
.99_{7} \\
.99_{9} \\
.99_{6} \\
.99_{8} \\
.99_{9}\end{array}$ \\
\hline Average_ & & & 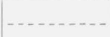 & $.99_{9}$ & $.99_{3}$ \\
\hline
\end{tabular}

a Thirty $\mathrm{ml}$ of benzene and 2 drops of 0.1-percent bromophthalein magenta $\mathrm{E}$ in benzene were added to the titration flask at the beginning of the titration. Acid and base were added to the solvent in approximately 1-ml increments and end-points were determined for the successive increments. The approximate calculated molarity of diphenylguanidinium diphenyl phosphate at the endpoint is given in the fourth column.

p Thirty ml of benzene and 2 drops of 0.1 -percent bromophthalein magenta $\mathrm{E}$ in benzene were mixed, and $3 \mathrm{ml}$ of this solution was added from a buret at the beginning of the experiment and before each succeeding end-point was determined. The concentration of diphenylguanidinium diphenyl phosphate was therefore $0.01-M$ at each end-point

the titration by the more cumbersome procedure used in experiment 2. Analogous results were obtained in titrating $0.02-M$ di-o-tolylguanidine with $0.02-M$ diphenyl phosphate by the same two procedures.

Table 5 presents examples of titrations of $0.02-M$ di-o-tolylguanidine with $0.02-M$ diphenyl phosphate in benzene using three different indicator dyes - an acidic indicator, bromophthalein magenta E, and two basic indicators, tamarack green (base) and Victoria blue B (base). The titration using bromophthalein magenta $\mathrm{E}$ was analogous to experiment 1 of table 4 and needs no further discussion. In the use of tamarack green (base) the initially vellow color becomes green when sufficient diphenyl phosphate has been added to combine with both ditolylguanidine and the indicator; the yellow color was restored by adding a little ditolylguanidine. In titrations with Victoria blue B (base) as the indicator, three distinct colors-orange, blue, and an intermediate gray-were observed. There is scarcely any difference in the results obtained with the three indicators, although on the whole we liked bromophthalein magenta $\mathrm{E}$ the best.
TABLE 5. Titrations of 0.02-M ditolylguanidine with $0.02-M$ diphenyl phosphate in benzene, using different indicators

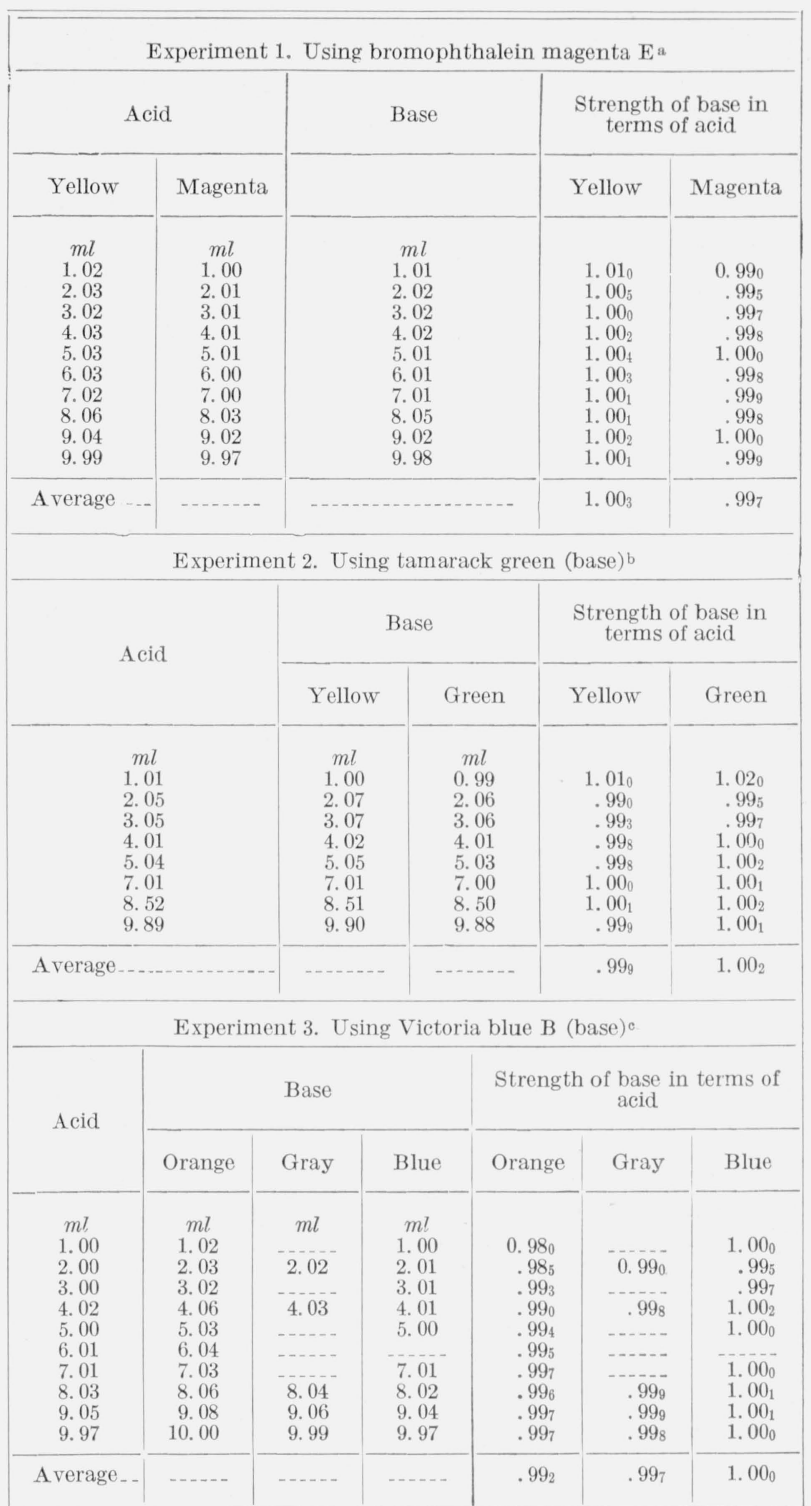

a Thirty $\mathrm{ml}$ of benzene and 2 drops of 0.1-percent bromophthalein magenta in benzene were added at the beginning of the titration.

b One drop of 0.1-percent tamarack green in benzene was added at the start. c Thirty $\mathrm{ml}$ of benzene and 2 drops of 0.1 -percent Victoria blue $\mathrm{B}$ in benzene were added at the beginning.

In addition to these titrations $0.02-M$ diphenylguanidine and $0.01-M$ di-o-tolylguanidine were titrated in benzene with diphenyl phosphate of equivalent concentration. The precision and accuracy were excellent, especially in view of the small volumes and low concentrations used. Diphenyl phosphate should be satisfactory for titrating other moderately strong bases in benzene and other aprotic solvents. It is probably a weaker acid than $p$-toluenesulfonic acid and other sulfonic acids, but is superior to the sulfonic acids in being more soluble in aprotic solvents and more easily obtained in a pure anhydrous state. 


\subsection{Concluding Remarks}

In the preceding sections it has been shown that accurate titrations with diphenyl phosphate can be performed in solvents as different as benzene and water, as well as in solvents of intermediate character. In view of its high acidic strength, its relatively high equivalent weight (250.19), the ease with which it can be obtained and maintained as anhydrous crystals, and its apparen i resistance to hydrolysis and alcoholysis at ordinary temperatures, it promises to be a useful material for acid-base titrations in both aqueous and nonaqueous solutions. More rigorous tests must be applied before it can be recommended as an acidimetric standard for work of the highest accuracy.

The properties of diphenyl phosphate suggest its use as an acid catalyst. Furthermore, it offers a means for extending knowledge of the behavior of acids. The great majority of the studies of acidic behavior made so far have been performed with aliphatic or aromatic carboxylic acids. However, studies of the sort illustrated in figures 3 and 4 show that generalizations reached through observations of the behavior of carboxylic acids in various solvents cannot safely be applied to all types of acids. The investigations with diphenyl phosphate are suggestive of interesting work still to be done with acids which as yet are not widely known, such as bisarylphosphonic acids.

The authors are indebted to James Stewart for measuring the benzene content of cyclohexane (table 1), and to the following companies for donating materials: American Cyanamid Company, for 5phenyl-9-diethyl Nile blue; Dow Chemical Company, for diphenyl phosphate; Heyden Chemical Corporation, for $o$-chlorobenzotrichloride.

\section{References}

[1] (a) M. M. Davis and P. J. Schuhmann, J. Research NBS 39, 221 (1947) RP1825.

(b) NBS Tech. News Bull. 36, 168 (1952).

[2] Dow Special Products, p. 88 (The Dow Chemical Company, Midland, Michigan, 1st edition, March 1939). See also the 1950 edition of this catalog.

[3] C. J. Penther and F. B. Rolfson, Ind. Eng. Chem., Anal. Ed. 15, 337 (1943).

[4] R. G. Bates and G. D. Pinching, J. Research NBS 43, 519 (1949) RP2043.

[5] A. Baeyer and V. Villiger, Ber. deut. chem. Ges. 37, 2848 (1904)

[6] K. Venkataraman, The chemistry of synthetic dyes, ch. XXIII (Academic Press, Inc., New York, N. Y., 1952).

[7] R. Meldola, J. Chem. Soc. (London) 41, 187 (1882).

[8] T. L. Hill, G. E. K. Branch, and M. Patapoff, J. Am. Chem. Soc. 6\%, 454 (1945).

[9] M. Nathansohn and P. Müller, Ber. deut. chem. Ges. 22, 1875 (1889)

[10] E. Noelting and J. Saas, Ber, deut. chem. Ges. 46, 952 (1913).
[11] I. M. Kolthoff, Acid-base indicators (The Macmillan Company, New York, N. Y., 1937; 4th German Edition, 1932, translated by C. Rosenblum).

[12] M. L. Crossley, R. J. Turner, C. M. Hofmann, P. F Dreisbach, and R. P. Parker, J. Am. Chem. Soc. 74, 578 (1952).

[13] (a) H. Hlasiwetz and A. Grabowski, Liebigs Ann. 139, 95 (1866); Zeitschrift für Chemie 9, 390 (1866).

(b) O. Rembold, Zeitschrift für Chemie 9, 651 (1866).

[14] L. Glutz, Liebigs Ann. 143, 181 (1867).

[15] G. Jacobsen, Ber. deut. chem. Ges. 8, 1519 (1875).

[16] M. Rapp, Liebigs Ann. 224, 156 (1884).

[17] W. Autenrieth, Ber. deut. chem. Ges. 30, 2369 (1897).

[18] J. M. A. Hoeflake, Rec. trav. chim. 36, 24 (1916).

[19] A. Bernton, Ber. deut. chem. Ges. 55B, 3361 (1922).

[20] F. Zetzsche and M. Nachmann, Helv. Chim. Acta 9, 705 (1926).

[21] S. Forssman, Biochem. Z 263, 135 (1933), through Chem. Abstracts 27, 4726 (1933) and Brit. Chem. Abstracts $1933 \mathrm{~A}, 897$.

[22] C. Morton, Quart. J. Pharm. Pharmacol. 3, 438 (1930).

[23] W. D. Kumler and J. J. Eiler, J. Am. Chem. Soc. 65, 2355 (1943)

[24] R. H. A. Plimmer and W. J. N. Burch, J. Chem. Soc. (London) 1929, 279.

[25] M. I. Smith and E. F. Stohlman, U. S. Public Health Repts. 48, 734 (1933).

[26] F. L. Breusch and H. Keskin, Rev, fac. sci. univ. Istanbul \%A, 182 (1942).

[27] E. Blumenthal and J. B. M. Herbert, Trans. Faraday Soc. 41, 611 (1945).

[28] (a) H. A. Pagel and F. W. McLafferty, Anal. Chem. 20, 272 (1948)

(b) H. A. Pagel, P. E. Toren, and F. W. MeLafferty, Anal. Chem. 21, 1150 (1949).

[29] C. S. Marvel, M. J. Copley, and E. Ginsberg, J. Am. Chem. Soc. 62, 3109 (1940).

[30] (a) W. Gordy and S. C. Stanford, J. Chem. Phys. 8, 170 (1940); 9, 204 (1941).

(b) W. Gordy, J. Chem. Phys. 9, 215 (1941)

(c) S. C. Stanford and W. Gordy, J. Am. Chem. Soc. 63, 1094 (1941)

[31] L. F. Audrieth and R. Steinman, J. Am. Chem. Soc. 63, 2115 (1941).

[32] (a) G. M. Kosolapoff and J. F. McCullough, J. Am. Chem. Soc. 73, 5392 (1951), and other papers by Kosolapoff and coworkers.

(b) G. M. Kosolapoff, Organophosphorus compounds (John Wiley and Sons, Inc., New York, N. Y., 1950).

[33] A. Hantzsch, Z. Elektrochem. 29, 221 (1923).

[34] (a) L. Lykken, P. Porter, H. D. Ruliffson, and F. D. Tuemmler, Ind. Eng. Chem., Anal. Ed. 16, 219 (1944).

(b) L. Lykken, Lubrication Eng. 2, 23, 64 (1946).

[35] S. Palit, Ind. Eng. Chem., Anal. Ed. 18, 246 (1946).

[36] (a) J. S. Fritz and N. M. Lisicki, Anal. Chem. 23, 589 (1951).

(b) J. S. Fritz, Acid-base titrations in nonaqueous solvents (G. Frederick Smith Chemical Company, Columbus, Ohio, 1952).

[37] M. M. Davis, P. J. Schuhmann, and M. E. Lovelace, J. Research NBS 41, 27 (1948) RP1900.

[38] H. Ferguson, Anal. Chem. 22, 289 (1950).

[39] M. M. Davis and H. B. Hetzer, J. Research NBS 46, 496 (1951) RP2219; 48, 381 (1952) RP2326.

[40] V. K. LaMer and H. C. Downes, J. Am. Chem. Soc. 53, 888 (1931)

[41] A. A. Maryott, J. Research NBS 38, 527 (1947) RP1794.

[42] (a) D. Vorländer, Ber. deut. chem. Ges. 36, 1485 (1903).

(b) D. Vorländer, with J. Fischer and F. Wildner, Ber. deut. chem. Ges. 66, 1789 (1933).

(c) D. Vorländer, Ber. deut. chem. Ges. 67, 145 (1934).

WAshington, January 20, 1955. 